\title{
GLOBAL HYPOELLIPTICITY AND LIOUVILLE NUMBERS ${ }^{1}$
}

\author{
STEPHEN J. GREENFIELD AND NOLAN R. WALLACH
}

ABSTRACT. We consider global hypoellipticity of constant coefficient differential operators on the 2-torus, and prove that it is equivalent to an algebraic growth condition on the symbol. This is applied to give necessary and sufficient conditions that a constant coefficient vector field be globally hypoelliptic. Similar results are true on compact homogeneous spaces.

Let $T^{2}=\left\{\left(\exp i \theta_{1}, \exp i \theta_{2}\right) ; \theta_{j} \in R\right\}$. If $L \in \mathfrak{D}^{\prime}\left(T^{2}\right)$ (a distribution on $T^{2}$ ) define $\hat{L}(n, m)=L\left(\exp \left(-\operatorname{in} \theta_{1}-i m \theta_{2}\right)\right)$ (see the normalization below for functions). $\mathcal{L}$ is a function on $Z \times Z$, the Fourier transform of $L$. We write $L \sim\{L(n, m)\}$ to indicate the correspondence. A function $T: Z \times Z$ $\rightarrow C$ is the Fourier transform of a distribution $L$ iff there is $K>0$ so that $|T(n, m)| \leqq K\left(n^{2}+m^{2}+1\right)^{K}$. ( $T$ is of polynomial growth ([S, Chapter 7]).) $L$ can be reconstructed from $T$ by:

$$
L=\sum_{n, m} T(n, m) \exp \left(i n \theta_{1}+i m \theta_{2}\right)
$$

(This implies the normalization

for $f$ a function.)

$$
f(g)=(2 \pi)^{-2} \int_{0}^{2 \pi} \int_{0}^{2 \pi} f(x, y) g(x, y) d x d y
$$

Suppose $P$ is an invariant differential operator on $T^{2}$ :

$$
P=\sum_{k, l=0}^{N} c_{k l} D_{1}^{k} D_{2}^{l},
$$

where $c_{k l} \in C$ and $D_{j}^{k}=\left(i^{-1} \partial / \partial \theta_{j}\right)^{k}$. Define $\hat{P}(n, m)=\sum_{k, l=0}^{N} c_{k l} n^{k} m^{l}$.

We say $P$ is globally hypoelliptic on $T^{2}$ when:

(GH) If $g \in C^{\infty}\left(T^{2}\right)$, and $P f=g, f \in D^{\prime}\left(T^{2}\right)$, then $f \in C^{\infty}\left(T^{2}\right)$.

THEOREM. $P$ is $(\mathrm{GH})$ if and only if there are positive real numbers $L, M$ so that:

(LM) $|\hat{P}(n, m)| \geqq L /\left(n^{2}+m^{2}\right)^{M}$, for $|n|,|m|$ sufficiently large.

Received by the editors November 5, 1970 and, in revised form, March 10, 1971.

AMS 1970 subject classifications. Primary 35H05, 42A92, 43A75.

Key words and phrases. Fourier coefficients, Liouville numbers, global hypoellipticity.

${ }^{1}$ Partially supported by NSF Grant numbers GP-20631 and GP-20647. 
Proof. When $h \in D^{\prime}\left(T^{2}\right)$, then $h \in C^{\infty}\left(T^{2}\right)$ iff

$$
\sup \frac{|\hat{h}(n, m)|}{\left(n^{2}+m^{2}+1\right)^{K}}<+\infty \text { for each } K \text {. }
$$

(This is equivalent to the perhaps better known condition

$$
\left.\sum_{|n|+|m|>0}|\hat{h}(n, m)|^{2}\left(n^{2}+m^{2}\right)^{K}<\infty .\right)
$$

Suppose now $P f=g, f, g \in D^{\prime}\left(T^{2}\right)$, and $f \sim\left\{a_{n m}\right\}$, and $g \sim\left\{b_{m n}\right\}$. Then $\hat{P}(n, m) a_{n m}=b_{n m}$.

$(\mathrm{LM}) \Rightarrow(\mathrm{GH})$ : By (LM), $\hat{P}(n, m) \neq 0$ if $|n|+|m|$ is sufficiently large. Thus $a_{n m}=\left(b_{n m} / \hat{P}(n, m)\right),|n|+|m|$ large. Since

$$
1 /|\hat{P}(n, m)| \leqq\left(n^{2}+m^{2}\right)^{M} / L,
$$

$\left({ }^{*}\right)$ for $\left\{b_{n m}\right\}$ gives $\left({ }^{*}\right)$ for $\left\{a_{n m}\right\}$.

$\sim(\mathrm{LM}) \Rightarrow \sim(\mathrm{GH})$ : If $(\mathrm{LM})$ is false, there is a sequence $\left\{\left(n_{j} ; m_{j}\right)\right\} \subseteq$ $Z \times Z$ so that $\left(n_{j} ; m_{j}\right) \rightarrow+\infty$ and $\left|\hat{P}\left(n_{j} ; m_{j}\right)\right| \leqq 1 /\left(n_{j}^{2}+m_{j}^{2}\right)^{j}$. Put $f=$ $\sum_{j} \exp \left(i n_{j} \theta_{1}+i m_{j} \theta_{2}\right)$. Then $f \in \mathfrak{D}^{\prime}\left(T^{2}\right)-C^{\infty}\left(T^{2}\right)$, but $P f \in C^{\infty}\left(T^{2}\right)$.

REMARK. If $P$ has order $N,|\hat{P}(n, m)| \leqq L\left(n^{2}+m^{2}\right)^{N / 2}$. If $P$ is elliptic, Gårding's inequality implies that we can take $M=-N / 2$ in (LM). We do not get local hypoellipticity on $T^{2}$ since we only consider simple behavior of the real Fourier transform $\hat{P}$ (see the criterion for hypoellipticity on $\boldsymbol{R}^{n}$ given in [H]. See also [B]).

Corollary. If $P$ is $(\mathrm{GH})$, then $P: C^{\infty}\left(T^{2}\right) \rightarrow C^{\infty}\left(T^{2}\right)$ is Fredholm of index 0.

Proof. $\quad P$ is continuous. (LM) implies that $\{\hat{P}(n, m)\}$ is almost never 0 . Put $S=\{(n, m) \mid \hat{P}(n, m)=0\} . S$ is finite. If $f \in C^{\infty}\left(T^{2}\right)$, and $f \sim\left\{a_{n m}\right\}$, let $\pi_{S} f$ be the $C^{\infty}$ function defined by $\left\{d_{n m}\right\}$, with $d_{n m}=a_{n m}$ for $(n, m) \in S$, and $d_{n, m}=0$ otherwise. Since $S$ is finite $\pi_{S}$ is a continuous projection.

ker $P=\left\{f \in C^{\infty}\left(T^{2}\right):\left(I-\pi_{S}\right) f=0\right\}$. If $P f=g$, and $g \in C^{\infty}\left(T^{2}\right)$ with $\pi_{S}(g)=0$, then there is $f \in C^{\infty}\left(T^{2}\right)$ so that $P f=g$ (obtain $f$ by Fourier transform from $g$-condition (LM) guarantees solvability). Thus dim ker $P$ $=\operatorname{dim}$ coker $P=$ cardinality of $S$.

Condition (LM) is rather particular. Suppose $P=D_{1}+c D_{2}, c=$ $a+i b \in C$. If $b \neq 0, P$ is elliptic and surely (GH). Suppose $b=0$, and $a \neq 0$.

If $a=R / S(R, S$ integers $)$, then $\hat{P}(-R t, S t)=0$. Thus $\hat{P}$ has infinitely many zeros, and $P$ is not (GH).

Proposition. Suppose $\alpha$ is a real irrational number. The vector field $P=D_{1}-\alpha D_{2}$ is globally hypoelliptic if and only if $\alpha$ is not a Liouville number. 
Proof. We recall (see [HW]) that $\alpha \in \boldsymbol{R}$ is a Liouville number if it can be approximated by rationals to any order. That is, for every positive integer $N$, there is $K>0$, and infinitely many integer pairs $(n, m)$ so that: $\left({ }^{* *}\right)|\alpha-n / m|<K / m^{N}$.

Condition (LM) for $P$ becomes: $|n-\alpha m| \geqq L /\left(n^{2}+m^{2}\right)^{M}$. Or $|\alpha-n / m| \geqq L /\left(n^{2}+m^{2}\right)^{M} m$. By adjusting $L$ we can suppose $L /\left(n^{2}+m^{2}\right)^{M} m<1$ for $|n|+|m|>0$. We need only consider $|\alpha-n / m|<1$. So $n$ and $m$ have the same order, and condition (LM) (with suitable change of $L$ ) becomes: $|\alpha-n / m| \geqq L / m^{2 M+1}$, for some $M, L>0$.

It is now clear that (LM) is equivalent to $\alpha$ not a Liouville number.

REMARK. Thus we have vector fields which are globally hypoelliptic but (since they are real vector fields) certainly not locally hypoelliptic. They are connected with the closed divergences of C. S. Herz [Z].

\section{BIBLIOGRAPHY}

B. R. Strichartz, Invariant pseudo-differential operators on a Lie group (to appear).

F. A. Cerezo and R. Rouvière, Solution élémentaire d'un opérateur différential linéaire invariant à gauche sur un groupe de Lie réal compact et sur un espace homogène réductif compact, Ann. Sci. École. Norm. Sup. (4) 2 (1969), 561-581.

H. L. Hörmander, Linear partial differential operators, Die Grundlehren der math. Wissenschaften, Band 116, Academic Press, New York; Springer-Verlag, Berlin, 1963. MR 28 \#4221.

HW. G. H. Hardy and E. M. Wright, An introduction to the theory of numbers, 4th ed., Oxford Univ. Press, London, 1960. MR 16, 673.

S. L. Schwartz, Théorie des distributions. Tomes I, II, Actualités Sci. Indust., nos. 1091, 1122, Hermann, Paris, 1950, 1951. MR 12, 31; 833.

W. Nolan R. Wallach, Differential operators on homogeneous spaces, Marcel Dekker, New York (to appear).

Z. C. Z. Herz, Functions which are divergences, Amer. J. Math. 92 (1970), 641-656.

Department of Mathematics, Rutgers University, New Brunswick, New JERSEY 08903 